\title{
A high-density genetic map developed by specific-locus amplified fragment (SLAF) sequencing and identification of a locus controlling anthocyanin pigmentation in stalk of Zicaitai (Brassica rapa L. ssp. chinensis var. purpurea)
}

Gui-Hua Li ${ }^{1,2}$, Han-Cai Chen ${ }^{1}$, Jia-Li Liu ${ }^{1,2}$, Wen-Long Luo ${ }^{1,2}$, Da-Sen Xie ${ }^{1,2}$, Shao-Bo Luo ${ }^{1,2}$, Ting-Quan Wu ${ }^{1,2}$, Waheed Akram ${ }^{1,2}$ and Yu-Juan Zhong ${ }^{1,2^{*}}$ (i)

\begin{abstract}
Background: Caixin and Zicaitai (Brassica rapa) belong to Southern and Central China respectively. Zicaitai contains high amount of anthocyanin in leaf and stalk resulting to the purple color. Stalk is the major edible part and stalk color is an economically important trait for the two vegetables. The aim of this study is to construct a high density genetic map using the specific length amplified fragment sequencing (SLAF-seq) technique to explore genetic basis for anthocyanin pigmentation traits via quantitative trait loci (QTL) mapping.

Results: We constructed a high generation linkage map with a mapping panel of F2 populations derived from 150 individuals of parental lines "Xianghongtai 01" and "Yinong 50D" with purple and green stalk respectively. The map was constructed containing 4253 loci, representing 10,940 single nucleotide polymorphism (SNP) markers spanning 1030.04 centiMorgans (CM) over 10 linkage groups (LGs), with an average distance between markers of $0.27 \mathrm{cM}$. Quantitative trait loci (QTL) analysis revealed that a major locus on chromosome 7 and 4 minor QTLs explaining 2.69-61.21\% of phenotypic variation (PVE) were strongly responsible for variation in stalk color trait. Bioinformatics analysis of the major locus identified 62 protein-coding genes. Among the major locus, there were no biosynthetic genes related to anthocyanin. However, there were several transcription factors like helix-loop-helix (bHLH) bHLH, MYB in the locus. Seven predicted candidate genes were selected for the transcription level analysis. Only bHLH49 transcription factor, was significantly higher expressed in both stalks and young leaves of Xianghongtai01 than Yinong50D. An insertion and deletion (InDel) marker developed from deletion/insertion in the promoter region of bHLH49 showed significant correlation with the stalk color trait in the F2 population.

Conclusion: Using the constructed high-qualified linkage map, this study successfully identified QTLs for stalk color trait. The identified valuable markers and candidate genes for anthocyanin accumulation in stalk will provide useful information for molecular regulation of anthocyanin biosynthesis. Overall our findings will lay a foundation for functional gene cloning, marker-assisted selection (MAS) and molecular breeding of important economic traits in B. rapa.
\end{abstract}

Keywords: Brassica rapa, SLAF-seq, Stalk color, Genetic map, Candidate gene

\footnotetext{
* Correspondence: zhongyujuan@gdaas.cn

'Vegetable Research Institute, Guangdong Academy of Agricultural Sciences, Guangzhou 510640, People's Republic of China

${ }^{2}$ Guangdong Key Laboratory for New Technology Research of Vegetables,

Guangzhou 510640, People's Republic of China
}

(c) The Author(s). 2019 Open Access This article is distributed under the terms of the Creative Commons Attribution 4.0 International License (http://creativecommons.org/licenses/by/4.0/), which permits unrestricted use, distribution, and reproduction in any medium, provided you give appropriate credit to the original author(s) and the source, provide a link to the Creative Commons license, and indicate if changes were made. The Creative Commons Public Domain Dedication waiver (http://creativecommons.org/publicdomain/zero/1.0/) applies to the data made available in this article, unless otherwise stated. 


\section{Background}

Brassica rapa is an important Brassica species with a long history of cultivation possessing, diverse qualities and distinct morphological traits [1]. Caixin (Brassica rapa L. ssp. parachinensis $2 \mathrm{n}=2 \mathrm{x}=20$ ) and Zicaitai (Brassica rapa L. ssp. chinensis var. purpurea Bailey 2n $=2 \mathrm{x}=20$ ) are two vegetable crops with edible stalks. Caixin also known as Chinese flowering cabbage, and Zicaitai were originated in Southern and Central China respectively. Both are popular in China and supplied to other countries of Asia, Europe, and the America. Attention has been devoted to molecular breeding for improving economically important traits of Brassica vegetables such as visual appearance and flavor. It is important to understand the genetic basis of quality related traits in Brassica species to accelerate the breeding process for better quality.

The stalk color, bolting and flowering time are important quantitative traits of $B$. rapa for breeding programs. High-density linkage maps and quantitative trait loci (QTL) analysis are exceptionally valuable tools for marker assisted molecular breeding of B. rapa. These analysis facilitate the identification of molecular markers either closely linked or co-segregating with target traits and genotype detection [2, 3]. QTL mapping mainly depends on marker density and population size. High-density linkage map is an indispensable tool for fine-scale mapping of phenotypic traits of interest, candidate gene cloning, comparative genomic analysis and genome assembly. The earliest DNA marker-based linkage map of B. rapa was constructed with 280 RFLP markers using an F2 population of 95 plants covering 1850 cM [4]. Recently, a genetic map of 120 Chinese cabbage F2 individuals of 711 bins representing 3985 single nucleotide polymorphism (SNP) markers was constructed using restriction site-associated DNA sequencing (RAD-seq) [5]. Multiple markers developed by sequencing facilitate high-density genetic map construction and QTL analysis. The abundance and stability of SNP markers makes them more useful than other markers for this purpose [6-8]. Genomic sequences of $B$. rapa and B. oleracea and some subfamilies provide an important foundation for SNP marker development $[1,9,10]$.

Next-generation sequencing (NGS) technology greatly expedites large scale SNP marker discovery. Reduced representation genome sequencing (RRGS) is a rapid and cost-efficient method for high-throughput SNP genotyping $[11,12]$. Several RRGS methods have been developed based on NGS technology that reduce the complexity of genomes by involvement of restriction enzymes $[13,14]$. SLAF-seq is a recently developed high-throughput sequencing technique that reduces cost and complexity of high-quality reference genome libraries [15]. This technology has been successfully used for high-density linkage map construction in diverse species such as common carp [15], sesame [16], soybean [17, 18], mei [19], and cucumber [20, 21]. To date, RRGS has seldom been applied to identify sequence polymorphisms in B. rapa, with only one high density map reported for Chinese cabbage [5]. A low density map has been previously constructed for Zicaitai composed of 161 InDel markers [22]. Thus, it is necessary to construct high density genetic map for Zicaitai to perform agronomic traits mapping.

Stalk color is an important quality trait for Chinese flowering cabbage and since the stalk is the major edible portion of this plant. The purple stalk and stem of Chinese cabbage "Zicaitai", are rich in anthocyanin [23]. The biosynthetic pathway of the purple pigment anthocyanin is a conserved network in many plant species and is well characterized [24]. The enzymes of anthocyanin biosynthetic pathway have been identified in Brassica [25]. It has been reported that a heterotrimeric MYBbasic helix-loop-helix (bHLH) -WD40 protein (MBW) complex regulate anthocyanin biosynthesis [26-29]. The bHLH group of transcription factors regulating anthocyanin biosynthesis belong to the subfamily IIIf bHLHs [30]. These are further divided into two different clades, the TT8 and GL3 clades. TT8 clade includes AN1(Petunia), TT8 (Arabidopsis), and Mutabilis (snapdragon), and GL3 clade includes JAF13 (petunia), GL3/EGL3 (Arabidopsis) and $\mathrm{R}$ (maize) [31]. Thus, there is more than one bHLH transcription factor (TF) regulating anthocyanin biosynthesis in plants.

Genetic basis of purple stalk trait of Chinese cabbage has not been studied up till now to best of our knowledge. There are a few studies available on genetic mapping or homology-based identification of anthocyanin biosynthetic pathway related genes in $B$. rapa, $B$. oleracea and $B$. juncea which have been used as references for stalk anthocyanin-related gene cloning. In $B$. rapa, single genes for leaf anthocyanin pigmentation were mapped onto chromosome A09 [32], A07 [33], and A03 within a genomic region of $54.87 \mathrm{~kb}$ [34]. Zicaitai cultivar contains multiple genes for anthocyanin pigmentation [22]. Several genes related to anthocyanin accumulation have been identified using map-based cloning. The purple pigmentation gene of cauliflower $(B$. oleracea var botrytis) encoded an R2R3-MYB TF and led to anthocyanin accumulation in curds and other tissues [35]. An anthocyanin-rich gene conferring purple leaves was localized in $B$. napus corresponding to a $99-\mathrm{kb}$ region of chromosome A03 and a BnAPR2 gene encoding adenosine $5^{\prime}$-phosphosulfate reductase was found to be a promising candidate [36]. In B. oleracea, a purple leaf trait controlled by a single dominant gene was localized to a 280-kb region of chromosome A09 and a dihidroflavonol reductase was identified as a candidate gene [37]. 
Although the leaves are anthocyanin rich in these germplasms, the stalks and stems have white color, which indicates independent genetic control from leaf color.

In the current study, genetic mapping of the purple stalk trait was performed using F2 progeny of two $B$. rapa genotypes with a significant difference in stalk color. The SLAF-seq technique was used for construction of a high-density genetic map containing 10,940 SNP markers distributed into 10 linkage groups (LGs), and spanning $1030.04 \mathrm{cM}$. Genetic mapping of purple stalk color revealed one major and four minor QTLs, and a closely linked marker was developed which could be applied in MAS breeding in stalk-edible Brassica plants. Potential candidate genes for the major QTL provide insight into molecular mechanisms regulating anthocyanin biosynthesis in $B$. rapa stalks.

\section{Results}

Inheritance of stalk color of Xianghongtai01 with Yinong50D

Stalk color analysis was performed in two parents, F1 and F2 individuals derived from Xianghongtai $01 \times$ Yinong 50D as shown in Fig. 1. A total of 150 F2 individuals were generated from the hybridization of Xianghongtai 01 (purple stalk and stem, 4th grade) and Yinong 50D (green stalk and stem, 1st grade) (Fig. 1). F1 individuals showed 2nd grade color. F2 individuals showed 1st-4th grades with a continuous distribution (Fig. 1). Stalk and stem color in the F2 population followed a continuous distribution that indicates that this trait might be quantitative and the alleles conferring purple stalks might only derive from the maternal parent.

\section{Analysis of SLAF data and genotyping}

Based on SLAF library construction and high-throughput sequencing, a total of $353.57 \mathrm{M}$ paired-end reads were obtained. For the reads, the GC content was $42.47 \%$ and Q30 ratio was $90.21 \%$. In the maternal line (Xianghongtai 01), 10,100,084 reads representing 80,935 SLAFs with average depth of 39.4 were generated (Table 1). In the paternal line (Yinong 50D), 10,181,878 reads representing 87,833 SLAFs with average coverage of 40.75 were generated (Table 1 ). In the F2 population, $2,221,944$ reads representing 77,079 SLAFs with average depth of 9.89 were generated (Table 1).

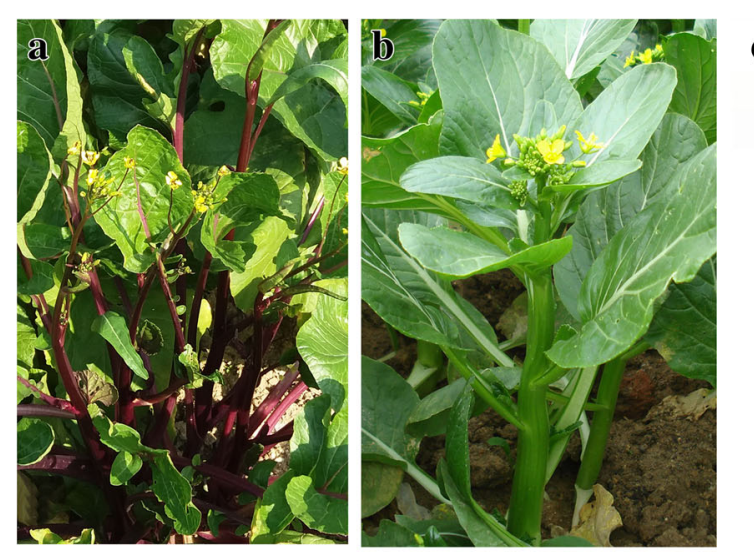

c
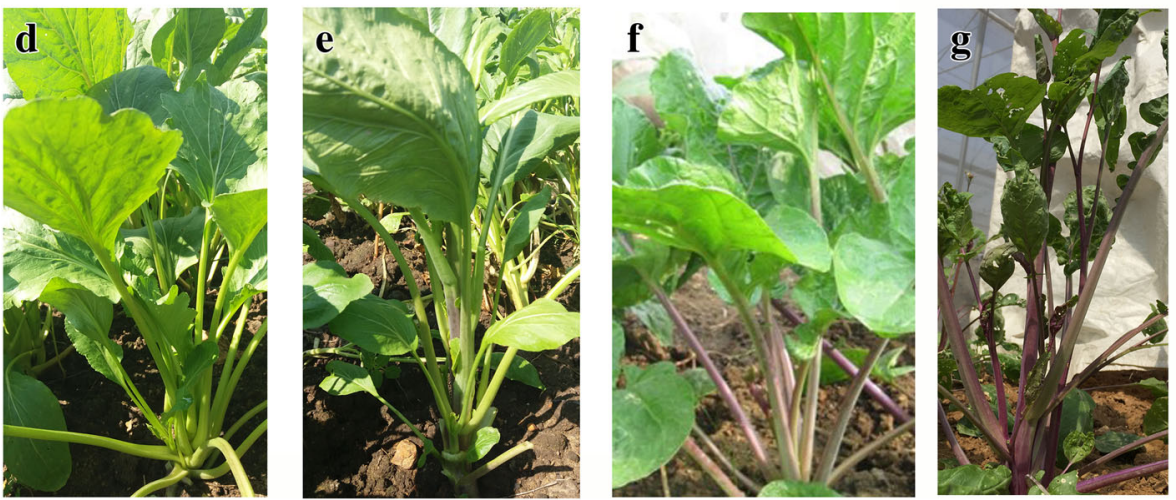

Fig. 1 The phenotypes of parental inbreds and F2 individuals and their frequency distributions, (a) maternal line, 'Xianghongtai01' (b) paternal line, 'Yinong50D', (c) the frequency distribution of the purple stalk trait in the F2 population, (d-g) phenotype of F2 individuals with 1st to 4st grades of stalk color 
Table 1 Summary of SLAF depths

\begin{tabular}{llll}
\hline Sample ID & SLAF number & Total depth & Average depth \\
\hline Yinong50D & 87,833 & $3,579,100$ & 40.75 \\
Xianghongtai01 & 80,935 & $3,188,840$ & 39.40 \\
Average of F2 & 77,079 & 762,464 & 9.89 \\
\hline
\end{tabular}

After filtration of low-depth SLAF tags, a total of 130,525 high-quality SLAFs were targeted with $20.3 \%$ $(26,557)$ polymorphic SLAFs. After screening out non-polymorphic and repetive markers the remaining 13,756 polymorphic SLAFs were classified into eight segregation patterns $(\mathrm{ab} \times \mathrm{cd}$, ef $\times \mathrm{eg}, \mathrm{hk} \times \mathrm{hk}, \mathrm{lm} \times \mathrm{ll}, \mathrm{nn} \times$ $\mathrm{np}$, aaxbb, abxcc, and $\mathrm{cc} \times \mathrm{ab}$ ) following the genotype encoding rule (Fig. 2). Finally, 10,112 of the 13,756 polymorphic SLAFs with aaxbb segregation pattern were selected for linkage map construction as Xianghongtai 01 and Yinong 50D are homozygous lines with genotypes of aa and bb (Fig. 2). Low quality SLAFs with parental sequence depth less than $10 \times$ were discarded. Finally, 4253 markers were used for linkage map construction after removing SLAFs with completeness less than $75 \%$ and significant segregation distortion $(\mathrm{p}-\mathrm{v}<0.05)$.

\section{Characteristics and evaluation of the high-density genetic} map

With a LOD (logarithm of odds ratio) threshold of 3.0, all 4253 markers were mapped onto ten linkage groups (LGs), designated LG1-LG10 using Highmap software (Table 2). The integrity of the mapped markers ranged from 99.15 to $100 \%$ which indicated high quality of the map (Table 2). The map spanned a total of $1030.04 \mathrm{cM}$ with an average interval between markers of $0.27 \mathrm{cM}$ and the largest interval of $14.99 \mathrm{cM}$. On average, LGs contained 425.3 markers that spanned an average distance of $103.00 \mathrm{cM}$. The genetic lengths of the $10 \mathrm{LGs}$ ranged from 73.12 (LG8) to $140.03 \mathrm{cM}$ (LG5). The LG with minimum number of markers, LG5, contained 235 SLAF markers while the largest, LG2, contained 677 markers. The average distance between markers ranged from $0.17 \mathrm{cM}$ (LG1, LG2) to $0.60 \mathrm{cM}$ (LG5) (Table 2). The locations of all markers in the genetic map were presented in Additional file 1.

The results showed that the SLAF markers in most LGs were well ordered. The double crossover should be at a low rate for high-density genetic map construction [38]. In this study, the percentage of double crossover of each LG is less than $0.04 \%$, which is in a permissible limit for high-density genetic map construction (Table 2). To uncover macro-colinearity between this map and the B. rapa genome (http://brassicadb.org/brad/), all 4253 markers on the final map were mapped onto 10 chromosomes in the genome (Fig. 3). The Spearman values of the 10 LGs ranged from 0.92 to 1.00 (Additional file 2). Most of the SLAF markers on the genetic map were in the same order as those in the corresponding chromosomes of the $B$. rapa genome.

\section{QTL analysis of purple stalk}

The maternal and paternal parents had purple and green stalk color, respectively. Based on the high-density

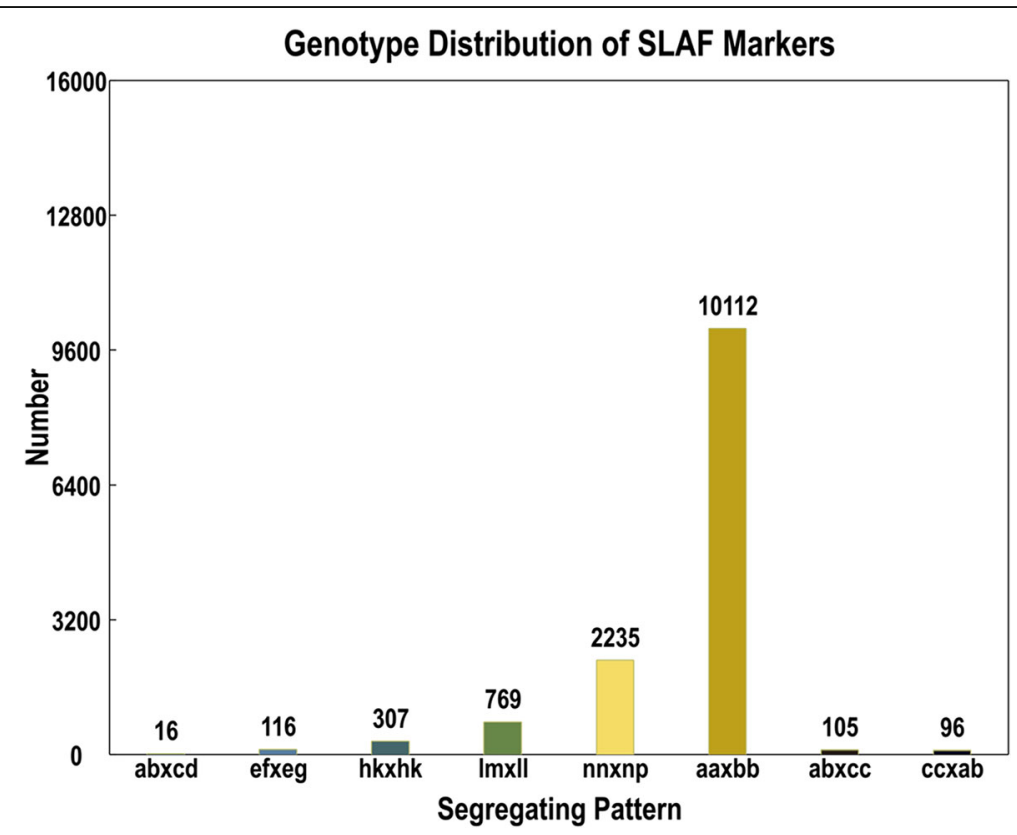

Fig. 2 Number of SLAF markers in each of eight segregation patterns 
Table 2 Basic information of the B. rapa genetic map

\begin{tabular}{llllllll}
\hline Linkage & Marker number & Map length (cM) & Max. distance (cM) & Marker interval (cM) & $\begin{array}{l}\text { Integrity } \\
\text { percentage (\%) }\end{array}$ & $\begin{array}{l}\text { Double crossover } \\
\text { percent (\%) }\end{array}$ & $\begin{array}{l}\text { Missing } \\
\text { percentage (\%) }\end{array}$ \\
\hline Chr 1 & 525 & 87.17 & 5.02 & 0.17 & 99.81 & 0.00 & 0.15 \\
Chr 2 & 677 & 118.36 & 3.20 & 0.17 & 100.00 & 0.01 & 0.35 \\
Chr 3 & 552 & 117.65 & 2.40 & 0.21 & 100.00 & 0.00 & 0.59 \\
Chr 4 & 284 & 82.96 & 2.40 & 0.29 & 100.00 & 0.02 & 0.27 \\
Chr 5 & 235 & 140.03 & 14.99 & 0.60 & 99.15 & 0.01 & 0.68 \\
Chr 6 & 411 & 109.65 & 3.47 & 0.27 & 100.00 & 0.04 & 0.33 \\
Chr 7 & 342 & 102.89 & 6.38 & 0.30 & 99.71 & 0.03 & 0.21 \\
Chr 8 & 354 & 73.12 & 3.62 & 0.21 & 100.00 & 0.01 & 0.28 \\
Chr 9 & 491 & 120.92 & 4.28 & 0.25 & 100.00 & 0.01 & 0.33 \\
Chr 10 & 382 & 77.29 & 3.67 & 0.20 & 100.00 & 0.04 & 0.31 \\
Total & 4253 & 1030.04 & 14.99 & 0.27 & 99.87 & &
\end{tabular}

genetic map and phenotypic characterization, QTL mapping of the stalk color trait were performed using Highmap with the Kosambi mapping function. Using joint analysis (LOD $\geq 3$ ), five QTLs associated with purple stalk of B. rapa (Brps) were detected on LG01 (qBrps1), LG07 (qBrps2), LG09 (qBrps3), and LG10 (qBrps4 and qBrps5), respectively (Table 3 and Fig. 4). Among all loci, qBrps 2 had the highest effect on purple color, explaining $61.2 \%$ of the phenotypic variation and mapping on to a region between Marker2181741 and Marker2262922, which spanned a genetic distance of $\sim 0.33 \mathrm{cM}$ and a physical distance of $\sim 0.38 \mathrm{Mb}$ (Table 3). Four additional minor QTLs were identified with PVE from 2.71 to $4.88 \%$, located on LG01, LG09 and LG10 (2), respectively. The sum of PVE values of the five QTLs was 77\%, indicating that the mapped QTLs explained most of the variance.
The locus analysis and candidate gene prediction

Based on the B. rapa genome (http://brassicadb.org/brad/ ) [1], 62 predicted protein-coding genes were identified in the small physical interval of qBrps $2(\sim 0.38 \mathrm{Mb}$ in length with 12 SLAF markers). According to results from Swissprot and BLASTX analysis, 59 of these 62 genes have been annotated (Additional file 3). None of these 59 genes correspond to anthocyanin biosynthetic genes in $B$ rapa [25]. However, we detected 7 genes that might regulate anthocyanin biosynthesis, encoding BIM2, MYB21, bHLH30, ARALYDRAFT (ARRI) and bHLH49 TFs; E3 ubiquitin-protein ligase (RINGB), and Alpha-xylosidase (Table 4). Bra004348 encoding a bHLH49 TF showed significantly higher expression in Xianghongtai 01 as compared to the Yinong 50D with 93 and 36 fold increases in young leaves and stalk samples, respectively (Fig. 5).

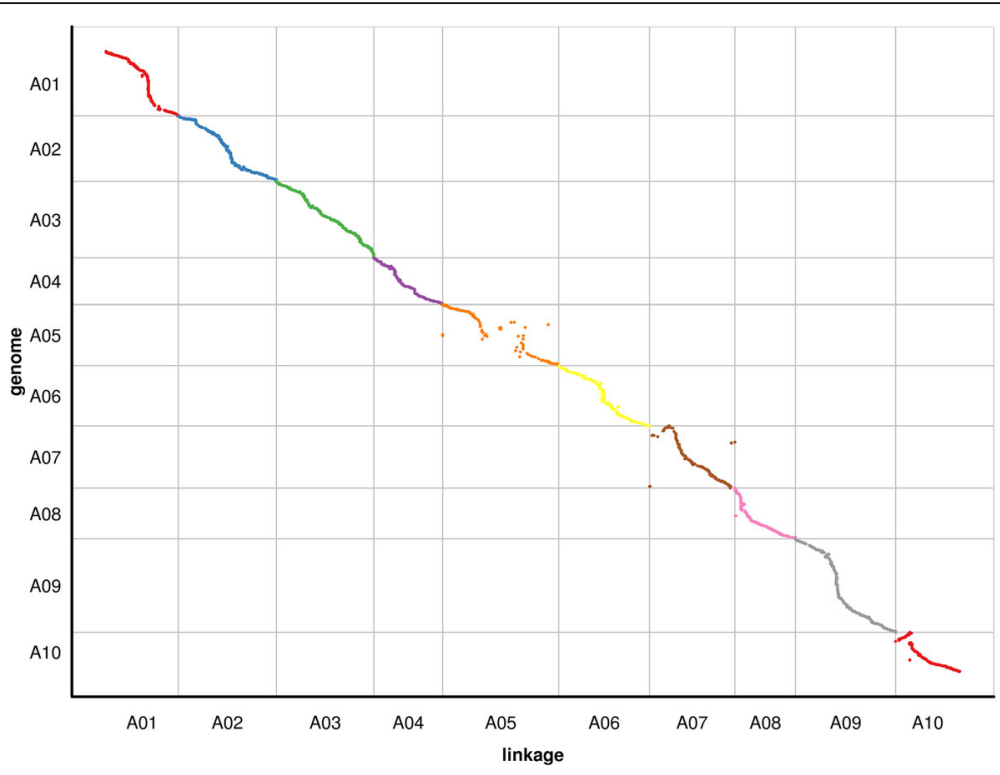

Fig. 3 Collinearity mapping of 10 linkage groups of B. rapa to the genomic sequence of $B$. rapa 
Table 3 All QTLS for stem color traits

\begin{tabular}{|c|c|c|c|c|c|c|c|c|}
\hline QTL name & Threshold & Chr & Start(cM) & End(cM) & Max LOD & ADD & DOM & PVE \\
\hline qBrps1 & 3 & $\mathrm{~A} 01$ & 25.74 & 26.07 & 3.94 & 0.15 & -0.58 & 2.80 \\
\hline qBrps2 & 3 & A07 & 78.54 & 78.87 & 34.30 & 2 & -0.79 & 61.21 \\
\hline aBrps3 & 3 & A09 & 33.02 & 33.36 & 5.18 & 0.63 & 0.09 & 5.90 \\
\hline qBrps4 & 3 & $\mathrm{~A} 10$ & 33.81 & 33.81 & 3.18 & 0.31 & 0.39 & 2.69 \\
\hline aBrps5 & 3 & $\mathrm{~A} 10$ & 37.14 & 62.96 & 4.71 & 0.50 & 0.18 & 3.93 \\
\hline
\end{tabular}

PVE phenotypic variance explained, Add additive value from xianghongtai01, Dom dominance value

\section{Linked InDel marker development and identification}

To confirm that linkage of $q$ Brps 2 with trait segregation, an InDel marker located in the promoter region was designed targeting a $7 \mathrm{bp}$ deletion in locus 1771 in the genome sequence of Bra004348 (Fig. 6). The InDel marker was polymorphic between the parents and were heterozygous in the F1, suggesting this marker might be associated with the purple stalk trait (Fig. 6). It was further used for screening the genotypes of 150 F2 plants with phenotypic data (Fig. 6). While InDel genotype could not distinguish among the grades of color based on this marker, all F2 individuals with the same genotype as P1 had purple stalks. There were seven individuals with inconsistency in genotype and phenotype for 1st and 2nd color grade F2 individuals and eight individuals with inconsistency in genotype and phenotype for 3rd and 4th grade F2 individuals. The effect of environment on the trait or the influence of the presence of other QTLs could contribute to misidentification of phenotype especially of 3th and 4th color grade F2 individuals. This InDel marker was further tested for genetic differentiation of the cultivars based on the stalk color. The cultivars having purple stalk trait showed the genotype as of Xianghongtai01, whereas, the cultivars with green stalk showed the genotype as of P2. Here the honglu hybrids with green stalk and F1 hybrid cultivar with light purple color at the bottom of the stalk showed the same genotype as of F1 (Fig. 6 and Additional file 4). These findings confirmed the association of this InDel marker with the phenotypic variation of the stalk color of different cultivars. The marker development confirmed that QTL analysis was reliable and supported the high accuracy of this map in QTL mapping of purple stalk.

\section{Discussion}

High-density genetic linkage maps play a significant role in trait mapping and map-based gene cloning

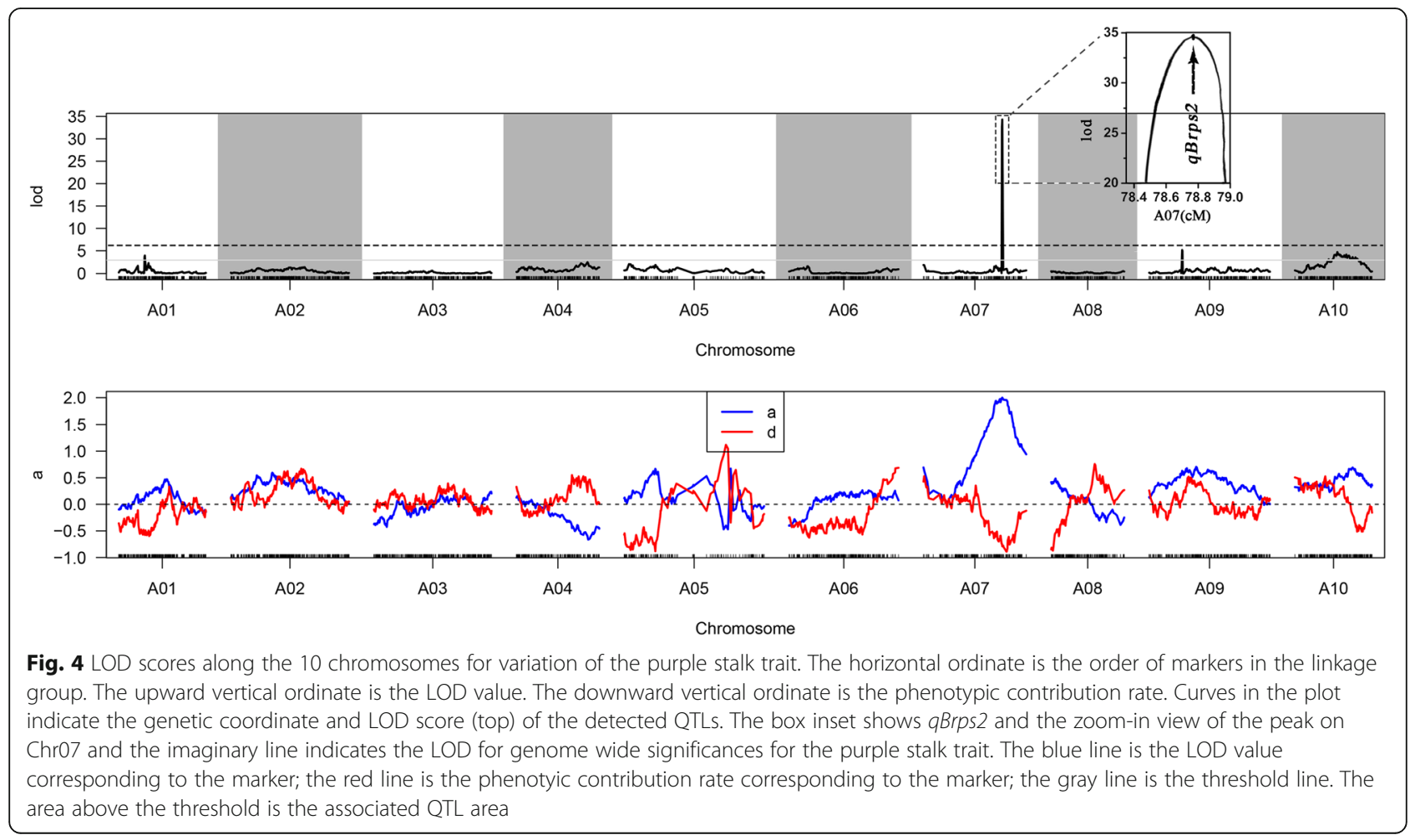


Table 4 Annotation of BrPs candidate genes

\begin{tabular}{llll}
\hline GeneID & Location & Direction & Swissprot_annotation \\
\hline Bra004355 & $21,462,654.21464299$ & + & Transcription factor BIM2 \\
Bra004297 & $21,105,151 . .21106182$ & + & Transcription factor MYB21 \\
Bra004338 & $21,375,472.21377053$ & + & Transcription factor bHLH30 \\
Bra004330 & $21,303,949 . .21305332$ & + & Probable transcription factor GLK1 \\
Bra004348 & $21,435,282 . .21437309$ & + & Transcription factor bHLH49 \\
Bra004339 & $21,382,039 . .21384773$ & + & Probable E3 ubiquitin-protein ligase LUL3 \\
Bra004319 & $21,235,196.21238948$ & - & Alpha-xylosidase 1 \\
\hline
\end{tabular}

[12, 39, 40]. With the development of NGS-based methods, high-throughput marker discovery and genotyping is easily achieved and has been successfully used in Brassica [5, 36, 37, 41-47]. The SLAF-seq strategy is a suitable method for large-scale SNP development and genotyping that combines high-throughput sequencing and locus-specific amplification [15, 46-48]. Several advantages including high success rate, accuracy, uniformity, stability, and low cost make SLAF-seq well suited for large-scale SNP marker discovery and genotyping [15].

In this study, SLAF sequencing technology was used in Chinese flowering cabbage and Zicaitai for genetic mapping of purple stalk color. We identified a total of 26,557 polymorphic SLAFs, and finally selected 4253 polymorphic markers with $75 \%$ of progeny for constructing the genetic map. The selection criterion is applicable for high-density genetic map construction (Table 2). The linear analysis of this genetic map and reference genome showed that the SLAF markers in most LGs were well ordered and the marker quality fulfilled the requirements for genetic map construction. There were only two big gaps $(15 \mathrm{cM}$ and $12 \mathrm{cM})$ between the genetic and physical distances on chromosomes A05 that was the longest linkage group. Coincidently, the genetic and physical inconsistences also occurred in this chromosome. Compared with another reported high density map of B. rapa, this map had fewer gaps and high linearity between genetic and physical distance [5]. While multiple genetic maps have been constructed for Chinese cabbage, there were only a few maps available for Chinese flowering cabbage. Guo used Zitaitai and Caixin as parents and constructed a linkage map using 161 InDel markers [22]. The map density was greatly improved in this study using SLAF-seq technology that facilitates genetic mapping of agronomic traits.

Regulation of anthocyanin accumulation is a complex process in Brassica plants. In previous studies it was seen that the purple characteristics were controlled by different single genes in B. oleracea and B. rapa germplasm with purple dominant to green [32-35]. In this study, purple stalk color was demonstrated to be a quantitative trait. It was similar to purple characteristics in leaves of an F2 population also derived from Zicaitai and Caixin [22]. However, we found that F2 individuals with 2nd color grade purple stalks have green leaves and the purple color is focused on the stalk and stem in one of the parent (Zicaitai). The fact that stalk color has different genetic control from leaf color indicates that the regulation of anthocyanin accumulation in plants is regulated by multiple genes. The identification of responsible genes by genetic mapping can facilitate understanding of the molecular mechanism of anthocyanin biosynthesis.

In previous studies, three loci were reported to control anthocyanin pigmentation [32-35]. Moreover, two QTLs for anthocyanin accumulation in leaves were identified: one major QTL on chr09 explained $56.7 \%$ of phenotypic variation; a minor QTL on chr07 had PVE of 16.3\% [22]. The physical region of the chr07 QTL was from $19,018,694$ to $24,359,626$ (5.3 Mb in internal length). While there were several QTLs for purple color or anthocyanin content in leaves in Brassica species. Here no QTLs conferring anthocyanin content in stalks have been previously reported. In this study, the QTL analysis of stalk color is performed since the stalk is the major edible part of Caixin and Zicaitai. Interestingly, a major QTL on chr 07 explaining 61.21\% of phenotypic variation was identified that was different from the major QTL for leaf anthocyanin contents. Minor QTLs on chr01, chr09 and chr10 were also identified with threshold of LOD $>3.0$, with PVE from 2.71 to $5.62 \%$. The major locus on chr07 from $21,101,820$ to $21,485,656$ (383.8 $\mathrm{kb}$ in internal length) was included in the reported minor QTL on this chromosome [22]. The distance here is much smaller than reported which suggests that the gene identified in this study might be controlling leaf anthocyanin contents. These results suggest that the high-density genetic map generated in this study can be successfully applied in QTL mapping.

The major locus in our study is mapped to an interval of $0.33 \mathrm{cM}$ in genetic size and $0.38 \mathrm{Mb}$ in physical size. The region contained only 62 inferred genes with 59 annotated. The small size of the region facilitated 

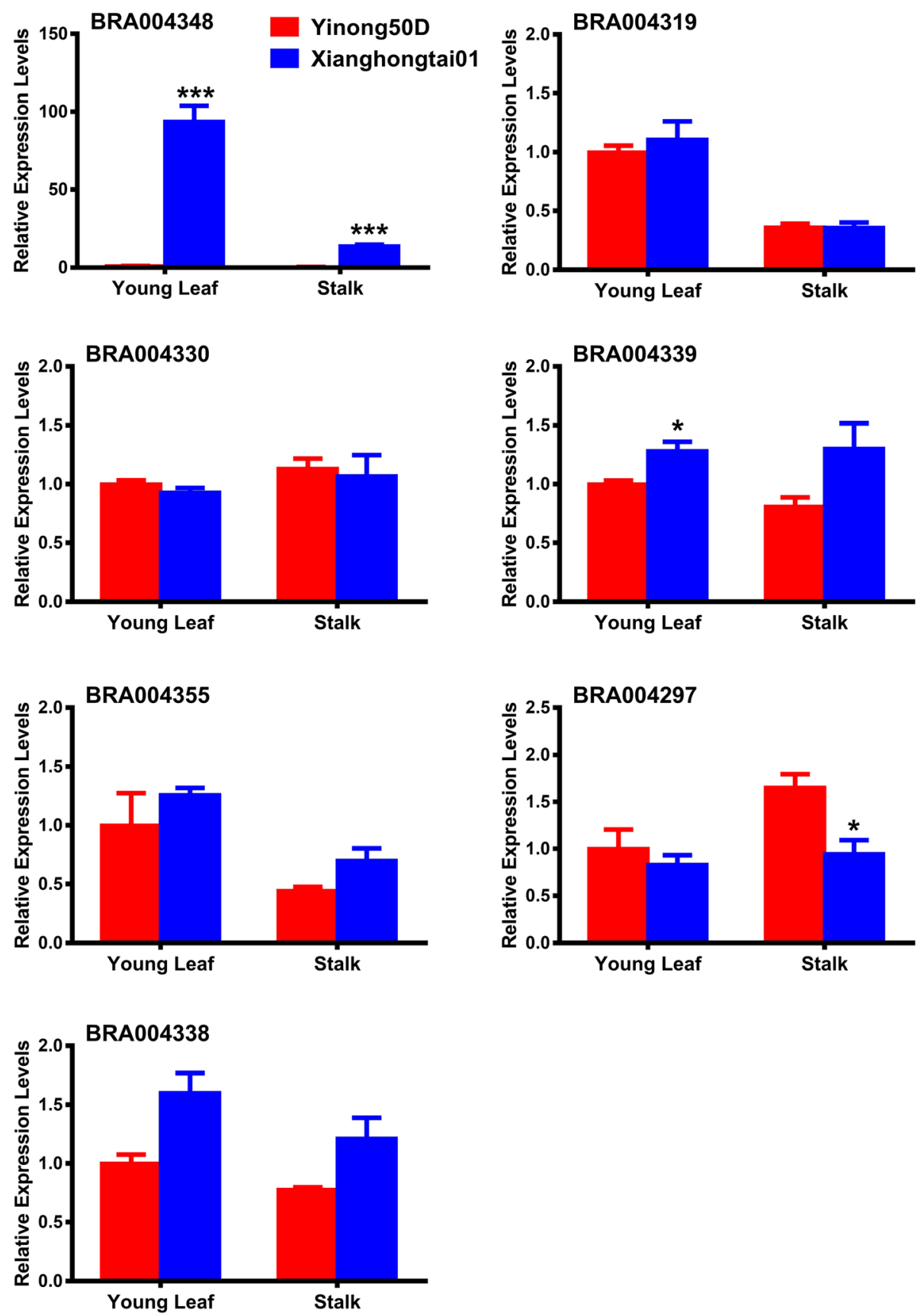

Fig. 5 Real-time PCR identification of selected candidate genes related to anthocyanin biosynthesis. There were seven candidate genes including five transcription factors which might regulate anthocyanin, BIM2, MYB21, bHLH3O, ARALYDRAFT (ARRI) and bHLH49; and E3 ubiquitin-protein ligase and Alpha-xylosidase. Significant differences of Xianghongtai01 compared withYinong50D are indicated by * based on the Students $t$ test, $\left({ }^{*} P<0.05,{ }^{*} P<0.01 ;{ }^{* *} P<0.001\right)$. The transcript levels of the genes are normalized to actin transcript level and expressed relative to PPIS which is set to 1 . In all cases means \pm SD $(n=3)$ are represented

candidate gene identification. Anthocyanin biosynthesis variation associated with this region may result from transcriptional regulation of structural genes since no salient structural genes were found in the locus. There were two $b H L H$ and one $M Y B T F$ gene(s) were identified in that locus. Seven possible candidate genes including these three transcription factor genes were selected for transcript level analysis. Only BrbHLH49 showed significantly higher expression in Xianghongtai 01 (purple stalk) than Yinong 50D (green stalk) in both young leaf and stalk tissues. Among the $133 \mathrm{bHLH}$ genes in Arabidopsis, there were at least four genes confirmed to be 
$\mathbf{a}$

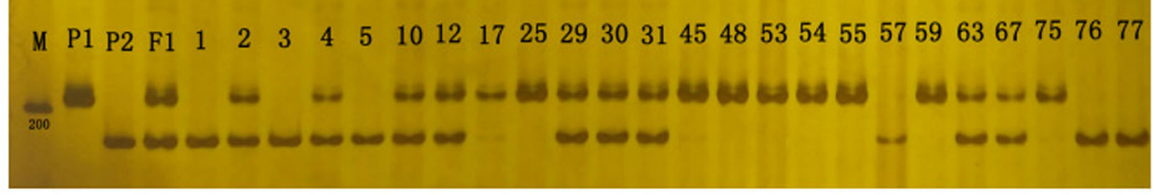

b
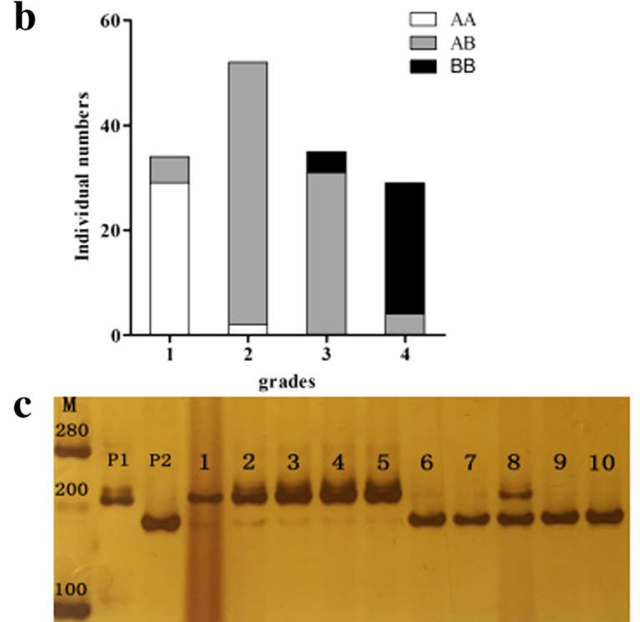

Fig. 6 Frequency distribution of the 150 F2 individuals based on the genotype of the InDel marker. a Gel image of InDel marker in parents and F2 individuals compared with 1 kb DNA marker, (b) Genotype and phenotype consistency analysis, (c) Gel image of InDel marker in parents and cultivars compared with 2 kb DNA marker (1: Hong hybrid 60, 2: Huahong 2; 3: Shiyuehong; 4: Xianghong12; 5: Xiangzaotai; 6: Biqin, 7: Youlu; 8: Bilu; 9: Honglu hybrid; 10: Youqing49)

related to anthocyanin biosynthesis. TT8 is the key regulator of anthocyanin and proanthocyanin biosynthesis [49]. The other two bHLH TFs, ENHANCER OF GLABRA3 (EGL3) and GLABRA3 (GL3) enhance anthocyanin biosynthesis together with PAP1 and PAP2. Another bHLH TF (PIF3), directly binds with the promoters of genes involved in the biosynthesis of anthocyanin and act as a positive component in PHYA-mediated anthocyanin biosynthesis [50]. In tomato, bHLH TF gene SlGL3 is also not participating in the putative MBW protein complex in tomato as is the case in Arabidopsis [51]. In maize, several bHLH TFs were identified to induce tissue-specific anthocyanin biosynthesis, such as $L c, R, B, S n$ and Hopi genes [52]. In carrot and ornamental cabbage, transcriptome analysis revealed there were several bHLH TFs were identified for purple pigmentation including bHLH137-like and bHLH168-like [53, 54]. Based on previous studies, bHLH TFs regulate anthocyanin biosynthesis through different mechanism and they might be tissue-specific to regulate anthocyanin accumulation. It is suggested that multiple bHLH TFs can be involved in anthocyanin accumulation in B. rapa. These TFs are either tissue specific or induced by the environment. The difference of major QTLs in the leaves and stalk regions suggests that bHLH TFs activity can be of tissue specific during anthocyanin biosynthesis in Zicaitai. Moreover, the members of the MBW complex, such as bHLH and MYBs, are modulated by environment stimuli [55]. However, the molecular mechanism for MBW components regulating anthocyanin biosynthesis in response to environmental stimuli is largely unknown [56]. In tomato, a bHLH TF AH was identified to be involved in anthocyanin biosynthesis that is developmentally regulated and induced by low temperature [57]. In apple, the molecular mechanism by which $M d b H L H 3$ regulates low temperature induced anthocyanin accumulation was identified [58]. The biosynthesis of anthocyanin in Zicaitai might be induced by low temperature since it was grown in winter in China and high temperature can inhibit the stalk anthocyanin accumulation. In previous study, the homologous EGL3 and GL3 located on chr09 were candidate genes for leaf anthocyanin accumulation in Zicaitai [22]. In the present study, we found that purple stalk color was not linked with change of leaf color, suggesting the two traits could be mainly regulated by two different genes which might be tissue specific. In the present study, BrbHLH49 on chr07 seems to be the best candidate gene for purple stalk color since the regulation of anthocyanin biosynthesis in Xianghongtai 01 is likely due to the high expression of this gene. The environmental stimuli involved in the regulation of anthocyanin biosynthesis has not yet been found. The regulation mechanisms 
of anthocyanin biosynthesis in leaves and stalks of Zicaitai remain unknown.

The linked InDel marker developed from the mutation of BrbHLH49 confirmed that variation of this gene is mainly linked with stalk color in F2 individuals and $B$. rapa cultivars. These results implicate that the BrbHLH49 was the most interesting candidate gene for increased anthocyanin biosynthesis in the stalk of Xianghongtai 01. Moreover, the linked InDel marker developed in this study could be used for molecular marker-assisted selection. Further analysis to identify candidate gene function and the genes responsible for anthocyanin biosynthesis needed to clarify the genetic mechanism underling anthocyanin accumulation in the stalk of Zicaitai.

\section{Conclusion}

In this study, we used SLAF-seq method to construct a high-genetic map with the most markers for $B$. rapa. The map was used to identify QTLs associated with stalk color. One major and four minor QTLs for stalk color were firstly identified. The major QTL explains $61.21 \%$ of phenotypic variation in which 62 genes were identified. In addition, 7 genes were considered to be potential genes in further study especially one bHLH transcription factor with obvious different expression in parents. Besides, we provide the valuable markers for stalk color. In a while, our findings will be of great use for MAS breeding of B. rapa and provide significant information for accurate QTL location of stalk anthocyanin accumulation.

\section{Methods}

Plant materials, phenotyping and DNA extraction

Two highly inbred lines of Chinese flowering cabbage, "Xianghongtai 01" with purple stalk and "Yinong 50D" having green stalk were used in this study. Xianghongtai 01 originated in Middle China whereas Yinong 50D from South China. These two inbred lines were generated after several generations of self-pollination and seeds were developed and owned by our lab. About 40 parental lines, $20 \mathrm{~F} 1$ individuals, $150 \mathrm{~F} 2$ individuals and 10 Zicaitai and Caixin cultivars were used in this study. Three-week old seedlings of the inbred lines and individuals were planted in the experimental field of the Vegetable Research Institute, Guangdong Academy of Agricultural Sciences, Guangzhou, China in November of 2016. Seedlings were planted in the ridges of hilled rows $(30 \mathrm{~cm}$ in height; $200 \mathrm{~cm}$ in width; $20 \mathrm{~cm}$ in distance of rows and columns). Stalk color was observed three times from 20 December 2016 to 10 January 2017 using 4 grades as follows: leaf stalk and stem were all green like the paternal parent, Yinong 50D, which was scored as 1st grade; leaf stalk and stem were all purple like the maternal parent, Xianghongtai 01, which was scored as 4th grade; and intermediate types with less to more purple leaf stalk and stem were scored as 2nd and 3rd grades. DNA was extracted from parental lines, F2 individuals and cultivars. Young leaves were frozen in liquid nitrogen immediately after harvest and stored at $-80^{\circ} \mathrm{C}$. Total genomic DNA was extracted using Cetyltrimethyl ammonium bromide (CTAB) method [59]. DNA quality was checked by NanoDrop 2000C spectrophotometer (Thermo Scientific, USA), and used for library construction.

\section{SLAF sequencing}

Specific-locus amplified fragment sequencing was performed on the DNA of parents and $150 \mathrm{~F}_{2}$ individuals as described by Sun with minor modifications [15]. A pilot experiment was performed to evaluate the restriction enzymes and conditions to obtain high-quality SLAFs. After evaluation, RsaI and HaeIII (NEB) were chosen for genomic DNA digestion. Afterwards, dual-index sequencing adapters were ligated to the fragments by T4 ligase (NEB). A polymerase chain reaction (PCR) was performed and amplicons ranging from 364 to $464 \mathrm{bp}$ (with indexes and adaptors) in size were targeted for purification using a Gel Extraction Kit (Qiagen, Hilden, Germany). These fragments were again amplified by PCR to be used for SLAF sequencing. Sequencing was performed using an Illumina HiSeq 2500 system (Illumina, Inc., San Diego, CA, USA) according to the manufacturer's recommendations at the Biomarker Technologies Corporation (Beijing, China).

\section{SLAF-seq data grouping and genotyping}

The SLAF-seq data grouping and genotyping was performed according to procedures described by Sun [15]. All SLAF reads were clustered according to sequence similarity after performing BLAST analysis [60]. Sequences with more than 95\% homology were grouped in one SLAF locus [15]. SNP loci in each SLAF locus were then detected among parental inbred lines. Minor allele frequency evaluation was performed to define alleles in each SLAF. Loci containing more than four tags were filtered out as possible repetitive SLAFs. Low-depth SLAFs (sequence depth of less than 10) were filtered out for downstream analysis. Potential markers that were defined as polymorphic SLAFs containing 2 4 tags were classified into eight segregation patterns as follows: $a b \times c d$, ef $\times$ eg, hk $\times$ hk, $l m \times l l, ~ n n \times n p, a a \times b b, a b \times c c$, and $\mathrm{cc} \times \mathrm{ab}$ ). Only the SLAF markers with the pattern of aaxbb were used for genetic map construction since F2 individuals were derived from two fully homozygous parental lines. 


\section{Linkage map construction and QTL mapping of stalk color}

HighMap software was used to order SLAF markers and for correction of genotyping errors within LGs [38]. The software constructs high-quality genetic maps based on an efficient maximum likelihood estimation method. All high quality SLAF markers were allocated to one of the ten LGs based on the location on the chromosome. The Kosambi mapping function was used to transform recombination percentages to genetic distances in cM. Subsequently, we applied the haplotype map and heat map to evaluate the quality of map [61]. "draw_haplotype-map.pl" was used to construct haplotype map, and "draw_heatmap.pl" was used to construct heat map, both of which were programmed by Beijing Biomarker Technologies Corporation. QTL analysis was performed using R/qtl software. Automatic cofactor selection (backward elimination, $P<0.05)$ was used for the detection of significantly associated markers as cofactors. LOD significance threshold levels were determined on the basis of 1000 permutations at significance levels of $P<0.05$. The location of each QTL was determined according to its LOD peak location and surrounding region. The percentage of phenotypic variance explained by a QTL (R2) was estimated at the highest probability peak. Candidate gene annotation was performed by referring to genome annotations available for B. napus and B. rapa (http:// brassicadb.org/brad/). The function of the predicted genes was determined using the Swissprot database and BLASTX.

\section{Real-time PCR identification}

RNA extracted from young leaves and stalks from 3-week-old plants was treated with DNaseI (Invitrogen, Carlsbad, CA, USA) and reverse transcribed using oligo $\mathrm{dT}$ as primer and Superscript reverse transcriptase (Invitrogen, Carlsbad, CA). Actin was used as a reference gene for qRT-PCR analysis. cDNA concentrations in different samples were normalized based on amplification of actin. Primers for 7 potential genes regulating anthocyanin biosynthesis and actin are listed in Additional file 5. To examine the expression of the 8 genes, a PCR mix was prepared with $1 \mu \mathrm{L}$ cDNA samples as templates in the qRT-PCR assay in the presence of a SYBR Green PCR Master Mix (Invitrogen, USA) and gene-specific primers. The reactions were performed in a BIO-RAD Cycler IQ Multi-Color Real Time PCR Detection System (Bio-Rad, Hercules, CA, USA). The relative levels of the amplified mRNA were evaluated according to Livak and Schmittgen according to the $2^{-\Delta \Delta \mathrm{Ct}}$ method using actin gene for normalization [62]. Means of 3 different plant replicates were analyzed, using Student's $t$-tests to determine significant differences.

\section{InDel marker development}

An InDel marker located in 21,435,052 at chr07 was developed based on the deletions/insertions in the candidate gene of Bra004348. Primers for the InDel markers were designed using Primer 5 (http://www.premierbiosoft.com) and genome re-sequencing data. The primers are listed in Additional file 5. The InDel marker was used for polymorphism screening between the two parental lines, F1, 150 F2 individuals and 10 cultivars. PCR products were separated on an $8 \%$ polyacrylamide denaturing sequencing gel and visualized by silver nitrate staining.

\section{Additional files}

Additional file 1: QTL analysis of purple stalk. (PDF $1004 \mathrm{~kb}$ )

Additional file 2: Spearman factor of each linkage group. (PDF 91 kb)

Additional file 3: Annotation of the genes in the major QTL locus. (PDF 96 kb)

Additional file 4: Zicaitai and Caixin cultivars used for marker assisted selection (PDF 83 kb)

Additional file 5: Primers involved in this study. (PDF $94 \mathrm{~kb}$ )

\section{Abbreviations}

ARRI: ARALYDRAFT; Brps: Purple stalk trait of B. rapa; cM: centiMorgans; CTAB: Cetyltrimethyl ammonium bromide; EGL3: ENHANCER OF GLABRA3; GL3: GLABRA3; ISSR: Inter-simple sequence repeat; LG: Linkage group; LOD: (logarithm of odds ratio); MAF: Minor allele frequency; MAS: Markerassistant selection; MBW: Heterotrimeric MYB-basic helix-loop-helix (bHLH) -WD40 protein; NGS: Next-generation sequencing; PCR: Polymerase chain re action; PVE: Phenotypic variance explained; QTL: Quantitative trait loci; RADseq: Restriction site-associated DNA sequencing; RAPD: Random amplified polymorphic DNA; RINGB: E3 ubiquitin-protein ligase; RRGS: Reduced representation genome sequencing; SLAF: Specific-locus amplified fragments; SNP: Single-nucleotide polymorphism; SSR: Simple; TF: Transcription factor

\section{Acknowledgements}

We would like to acknowledge Prof. Zheng-Jie Wan for technical assistance.

\section{Funding}

This study was supported by the projects of the Guangdong Science and Technology Foundation (2015B070701014), the Guangzhou Science and Technology Foundation (201704020100), the projects of the Guangdong Science and Technology Foundation (2018A030313770). The funding bodies had no role in study design, data collection and analysis, interpretation of data, decision to publish, or writing the manuscript.

\section{Availability of data and materials}

The listed markers in the genetic map in our study are available in the Additional file 1.The sequences of markers and the raw datasets analyzed in the study are not publicly available but can be available, on reasonable request from the corresponding author.

\section{Authors' contributions}

YJZ and GHL conceived and designed the experiment and wrote the manuscript; GHL and HCC guided purple trait analysis, analyzed the map and QTL data; JLL and DSX collected leaf samples and extracted population DNA and analyzed phenotypes in field, WLL developed the markers and analyzed the linkage relationship with purple stalk trait, SBL and TQW performed the candidate gene analysis including real-time analysis, AW gave suggestion to the manuscript and revise the manuscript. All authors have read and approved the manuscript. 
Ethics approval and consent to participate

Not applicable.

\section{Consent for publication}

Not applicable.

\section{Competing interests}

The authors declare that they have no competing interests.

\section{Publisher's Note}

Springer Nature remains neutral with regard to jurisdictional claims in published maps and institutional affiliations.

\section{Received: 21 November 2018 Accepted: 15 April 2019}

\section{Published online: 07 May 2019}

\section{References}

1. Wang X, Wang H, Wang J, Sun R, Wu J, Liu S, Bai Y, Mun JH, Bancroft I, Cheng $F$, et al. The genome of the mesopolyploid crop species Brassica rapa. Nat Genet. 2011;43(10):1035-9.

2. Collard BCY, Jahufer MZZ, Brouwer JB, Pang ECK. An introduction to markers, quantitative trait loci (QTL) mapping and marker-assisted selection for crop improvement: the basic concepts. Euphytica. 2005;2005:169-96.

3. $\mathrm{Xu} \mathrm{Y,} \mathrm{Crouch} \mathrm{JH.} \mathrm{Marker-assisted} \mathrm{selection} \mathrm{in} \mathrm{plant} \mathrm{breeding:} \mathrm{from}$ publications to practice. Crop Sci. 2008:48(2):391-407.

4. Song KM, Suzuki JY, Slocum MK, Williams PM, Osborn TC. A linkage map of Brassica rapa (syn. campestris) based on restriction fragment length polymorphism loci. Tagtheoretical \& Applied Geneticstheoretische Und Angewandte Genetik. 1991;82(3):296-304.

5. Huang L, Yang Y, Zhang F, Cao J. A genome-wide SNP-based genetic map and QTL mapping for agronomic traits in Chinese cabbage. Sci Rep. 2017;7: 46305.

6. Wang N, Fang L, Xin H, Wang L, Li S. Construction of a high-density genetic map for grape using next generation restriction-site associated DNA sequencing. BMC Plant Biol. 2012;12(1):148.

7. Bourgeois YX, Lhuillier E, Cézard T, Bertrand JA, Delahaie B, Cornuault J, Duval T, Bouchez O, Milá B, Thébaud C. Mass production of SNP markers in a nonmodel passerine bird through RAD sequencing and contig mapping to the zebra finch genome. Mol Ecol Resour. 2013;13(5):899-907.

8. Fusari CM, Lia W, Hopp HE, Heinz RA, Paniego NB. Identification of single nucleotide polymorphisms and analysis of linkage disequilibrium in sunflower elite inbred lines using the candidate gene approach. BMC Plant Biol. 2008;8(1):7

9. Cheng F, Sun R, Hou X, Zheng H, Zhang F, Zhang Y, Liu B, Liang J, Zhuang M, Liu Y. Subgenome parallel selection is associated with morphotype diversification and convergent crop domestication in Brassica rapa and Brassica oleracea. Nat Genet. 2016:48(10):1218-24.

10. Liu S, Liu Y, Yang X, Tong C, Edwards D, Parkin IA, Zhao M, Ma J, Yu J, Huang $S$, et al. The Brassica oleracea genome reveals the asymmetrical evolution of polyploid genomes. Nat Commun. 2014;5:3930.

11. Van Tassell C, Smith T, Matukumalli L, Taylor J, Schnabel R, Lawley C, Haudenschild C, Moore S, Warren W. Ts: SNP discovery and allele frequency estimation by deep sequencing of reduced representation libraries. Nat Methods. 2008;5(3):247-52.

12. Hyten DL, Cannon SB, Song Q, Weeks N, Fickus EW, Shoemaker RC, Specht $J E$, Farmer AD, May GD, Cregan PB. High-throughput SNP discovery through deep resequencing of a reduced representation library to anchor and orient scaffolds in the soybean whole genome sequence. BMC Genomics. 2010;11(1):38.

13. Baxter SW, Davey JW, Johnston JS, Shelton AM, Heckel DG, Jiggins CD, Blaxter ML. Linkage mapping and comparative genomics using nextgeneration RAD sequencing of a non-model organism. PLoS One. 2011;6(4): e19315.

14. Pfender WF, Saha MC, Johnson EA, Slabaugh MB. Mapping with RAD (restriction-site associated DNA) markers to rapidly identify QTL for stem rust resistance in Lolium perenne. Theor Appl Genet. 2011;122(8):1467-80.

15. Sun X, Liu D, Zhang X, Li W, Liu H, Hong W, Jiang C, Guan N, Ma C, Zeng $H$, et al. SLAF-seq: an efficient method of large-scale De novo SNP discovery and genotyping using high-throughput sequencing. PLoS One. 2013;8(3):e58700
16. Zhang Y, Wang L, Xin H, Li D, Ma C, Xia D, Hong W, Zhang X. Construction of a high-density genetic map for sesame based on large scale marker development by specific length amplified fragment (SLAF) sequencing. BMC Plant Biol. 2013;13(1):1-12.

17. Li B, Tian L, Zhang J, Huang L, Han F, Yan S, Wang L, Zheng H, Sun J. Construction of a high-density genetic map based on large-scale markers developed by specific length amplified fragment sequencing (SLAF-seq) and its application to QTL analysis for isoflavone content in Glycine max. BMC Genomics. 2014;15(1):1086.

18. Qi Z, Huang L, Zhu R, Xin D, Liu C, Han X, Jiang H, Hong W, Hu G, Zheng H, et al. A high-density genetic map for soybean based on specific length amplified fragment sequencing. PLoS One. 2014;9(8):e104871.

19. Zhang J, Zhang Q, Cheng T, Yang W, Pan H, Zhong J, Huang L, Liu E. Highdensity genetic map construction and identification of a locus controlling weeping trait in an ornamental woody plant (Prunus mume Sieb. et Zucc). DNA Res. 2015;22(3):183.

20. Wei Q, Wang Y, Qin X, Zhang Y, Zhang Z, Wang J, Li J, Lou Q, Chen J. An SNP-based saturated genetic map and QTL analysis of fruit-related traits in cucumber using specific-length amplified fragment (SLAF) sequencing. BMC Genomics. 2014;15(1):1158.

21. Xu X, Xu R, Zhu B, Yu T, Qu W, Lu L, Xu Q, Qi X, Chen X. A high-density genetic map of cucumber derived from specific length amplified fragment sequencing (SLAF-seq). Front Plant Sci. 2014;5(768):768.

22. Guo N, Wu J, Zheng S, Cheng F, Liu B, Liang J, et al. Anthocyanin profile characterization and quantitative trait locus mapping in zicaitai (Brassica rapa L. ssp. chinensis var. purpurea). Mol Breed. 2015;35(5):113.

23. Podsędek A. Natural antioxidants and antioxidant capacity of Brassica vegetables: a review. LWT-Food Sci Technol. 2007;40(1):1-11.

24. Holton TA, Cornish EC. Genetics and biochemistry of anthocyanin biosynthesis. Plant Cell. 1995;7(7):1071.

25. Guo N, Cheng F, Wu J, Liu B, Zheng S, Liang J, Wang X. Anthocyanin biosynthetic genes in Brassica rapa. BMC Genomics. 2014;15:426.

26. Baudry A, Heim MA, Dubreucq B, Caboche M, Weisshaar B, Lepiniec L. TT2, TT8, and TTG1 synergistically specify the expression of BANYULS and proanthocyanidin biosynthesis in Arabidopsis thaliana. Plant J. 2004;39(3): 366-80.

27. Zhang F, Gonzalez A, Zhao M, Payne CT, Lloyd A. A network of redundant bHLH proteins functions in all TTG1-dependent pathways of Arabidopsis. Development. 2003;130(20):4859-69.

28. Zimmermann IM, Heim MA, Weisshaar B, Uhrig JF. Comprehensive identification of Arabidopsis thaliana MYB transcription factors interacting with R/B-like BHLH proteins. Plant J. 2004;40(1):22-34.

29. Xu W, Dubos C, Lepiniec L. Transcriptional control of flavonoid biosynthesis by MYB-bHLH-WDR complexes. Trends Plant Sci. 2015;20(3):176-85.

30. Heim MA, Jakoby M, Werber M, Martin C, Weisshaar B, Bailey PC. The basic helix-loop-helix transcription factor family in plants: a genome-wide study of protein structure and functional diversity. Mol Biol Evol. 2003;20(5):735-47.

31. Lim S-H, Kim D-H, Kim JK, Lee J-Y, Ha S-H. A radish basic helix-loop-helix transcription factor, RsTT8 acts a positive regulator for anthocyanin biosynthesis. Front Plant Sci. 2017:8:1917.

32. Burdzinski C, Wendell DL. Mapping the anthocyaninless (anl) locus in rapidcycling Brassica rapa (RBr) to linkage group R9. BMC Genet. 2007;8:64.

33. Hayashi K, Matsumoto S, Tsukazaki H, Kondo T, Kubo N, Hirai M. Mapping of a novel locus regulating anthocyanin pigmentation in Brassica rapa. Breed Sci. 2010;60(1):76-80.

34. Wang W, Zhang D, Yu S, Liu J, Wang D, Zhang F, Yu Y, Zhao X, Lu G, Su T. Mapping the BrPur gene for purple leaf color on linkage group A03 of Brassica rapa. Euphytica. 2014;199(3):293-302.

35. Chiu L-W, Zhou X, Burke S, Wu X, Prior RL, Li L. The purple cauliflower arises from activation of a MYB transcription factor. Plant Physiol. 2010;154(3): $1470-80$.

36. Li H, Zhu L, Yuan G, Heng S, Yi B, Ma C, Shen J, Tu J, Fu T, Wen J. Fine mapping and candidate gene analysis of an anthocyanin-rich gene, BnaA. PL1, conferring purple leaves in Brassica napus L. Mol Gen Genomics. 2016; 291(4):1523-34.

37. Liu X-p, Gao B-Z, Han F-q, Fang Z-Y, Yang L-m, Zhuang M, Lv H-h, Liu Y-M, Li Z-S, Cai C-c. Genetics and fine mapping of a purple leaf gene, BoPr, in ornamental kale (Brassica oleracea L. var. acephala). BMC Genomics. 2017;18(1):230.

38. Liu D, Ma C, Hong W, Huang L, Liu M, Liu H, Zeng H, Deng D, Xin H, Song J. Construction and analysis of high-density linkage map using highthroughput sequencing data. PLoS One. 2014;9(6):e98855. 
39. Shifman S, Bell JT, Copley RR, Taylor MS, Williams RW, Mott R, Flint J. A highresolution single nucleotide polymorphism genetic map of the mouse genome. PLoS Biol. 2006;4(12):e395.

40. West MA, Van LH, Kozik A, Kliebenstein DJ, Doerge RW, St Clair DA, Michelmore RW. High-density haplotyping with microarray-based expression and single feature polymorphism markers in Arabidopsis. Genome Res. 2006;16(6):787.

41. Trick M, Long Y, Meng J, Bancroft I. Single nucleotide polymorphism (SNP) discovery in the polyploid Brassica napus using Solexa transcriptome sequencing. Plant Biotechnol J. 2009;7(4):334-46.

42. Bus A, Hecht J, Huettel B, Reinhardt R, Stich B. High-throughput polymorphism detection and genotyping in Brassica napus using nextgeneration RAD sequencing. BMC Genomics. 2012;13(1):281.

43. Delourme R, Falentin C, Fomeju BF, Boillot M, Lassalle G, Andre I, Duarte J, Gauthier V, Lucante N, Marty A, et al. High-density SNP-based genetic map development and linkage disequilibrium assessment in Brassica napus $L$. BMC Genomics. 2013;14:120.

44. Chung $\mathrm{H}$, Jeong YM, Mun JH, Lee SS, Chung WH, Yu HJ. Construction of a genetic map based on high-throughput SNP genotyping and genetic mapping of a TuMV resistance locus in Brassica rapa. Mol Gen Genomics. 2014;289(2):149-60.

45. Geng X, Jiang C, Yang J, Wang L, Wu X, Wei W. Rapid identification of candidate genes for seed weight using the SLAF-Seq method in Brassica napus. PLoS One. 2016;11(1):e0147580.

46. Yu S, Su T, Zhi S, Zhang F, Wang W, Zhang D, Zhao X, Yu Y. Construction of a sequence-based bin map and mapping of QTLs for downy mildew resistance at four developmental stages in Chinese cabbage ( Brassica rapa L. ssp. pekinensis ). Mol Breed. 2016;36(4):1-12.

47. Zhou Q, Zhou C, Zheng W, Mason AS, Fan S, Wu C, Fu D, Huang Y. Genome-wide SNP markers based on SLAF-Seq uncover breeding traces in rapeseed (Brassica napus L.). Front Plant Sci. 2017;8:648.

48. Zhao Z, Gu H, Sheng X, Yu H, Wang J, Long H, Dan W. Genome-wide single-nucleotide polymorphisms discovery and high-density genetic map construction in cauliflower using specific-locus amplified fragment sequencing. Front Plant Sci. 2016;7(393).

49. Nesi N, Debeaujon I, Jond C, Pelletier G, Caboche M, Lepiniec L. The TT8 gene encodes a basic helix-loop-helix domain protein required for expression of DFR and BAN genes in Arabidopsis siliques. Plant Cell. 2000; 12:1863-78.

50. Shin J, Park E, Choi G. PIF3 regulates anthocyanin biosynthesis in an HY5dependent manner with both factors directly binding anthocyanin biosynthetic gene promoters in Arabidopsis. Plant J. 2007;49(6):981-94.

51. Tominaga-Wada R, Nukumizu Y, Wada T. Tomato (Solanum lycopersicum) homologs of TRIPTYCHON (SITRY) and GLABRA3 (SIGL3) are involved in anthocyanin accumulation. Plant Signal Behav. 2013;8(7):e24575.

52. Petroni K, Cominelli E, Consonni G, Gusmaroli G, Gavazzi G, Tonelli C. The developmental expression of the maize regulatory gene Hopi determines germination-dependent anthocyanin accumulation. Genetics. 2000;155(1): 323-36.

53. Kodama M, Brinch-Pedersen H, Sharma S, Holme IB, Joernsgaard B, Dzhanfezova T, Amby DB, Vieira FG, Liu S, Gilbert MTP. Identification of transcription factor genes involved in anthocyanin biosynthesis in carrot (Daucus carota L.) using RNA-Seq. BMC Genomics. 2018;19(1):811.

54. Jin S-W, Rahim MA, Afrin KS, Park J-I, Kang J-G, Nou I-S. Transcriptome profiling of two contrasting ornamental cabbage (Brassica oleracea var. acephala) lines provides insights into purple and white inner leaf pigmentation. BMC Genomics. 2018;19(1):797.

55. Lin-wang K, Micheletti D, Palmer J, Volz R, Lozano L, Espley R, Hellens RP, Chagne D, Rowan DD, Troggio M. High temperature reduces apple fruit colour via modulation of the anthocyanin regulatory complex. Plant Cell Environ. 2011;34(7):1176-90.

56. Gonzalez A. Pigment loss in response to the environment: a new role for the WD/bHLH/MYB anthocyanin regulatory complex. New Phytol. 2009; 182(1):1-3.

57. Qiu Z, Wang X, Gao J, Guo Y, Huang Z, Du Y. The tomato Hoffman's anthocyaninless gene encodes a bHLH transcription factor involved in anthocyanin biosynthesis that is developmentally regulated and induced by low temperatures. PLoS One. 2016;11(3):e0151067.

58. Xie XB, Li S, Zhang RF, Zhao J, Chen YC, Zhao Q, Yao YX, You CX, Zhang XS, Hao YJ. The bHLH transcription factor MdbHLH3 promotes anthocyanin accumulation and fruit colouration in response to low temperature in apples. Plant Cell Environ. 2012;35(11):1884-97.

59. Murray MG, Thompson WF. Rapid isolation of high weight plant DNA. Nucleic Acids Res. 1980:8(19):4321-5.

60. Kent WJ. BLAT—the BLAST-like alignment tool. Genome Res. 2002;12(4): 656-64.

61. Liu L, Qu C, Wittkop B, Yi B, Xiao Y, He Y, Snowdon RJ, Li J. A high-density SNP map for accurate mapping of seed fibre QTL in Brassica napus L. PLoS One. 2013;8(12):e83052.

62. Livak KJ, Schmittgen TD. Analysis of relative gene expression data using real-time quantitative PCR and the 2(-Delta Delta $C(T))$ method. Methods. 2012;25(4):402-8.

\section{Ready to submit your research? Choose BMC and benefit from:}

- fast, convenient online submission

- thorough peer review by experienced researchers in your field

- rapid publication on acceptance

- support for research data, including large and complex data types

- gold Open Access which fosters wider collaboration and increased citations

- maximum visibility for your research: over $100 \mathrm{M}$ website views per year

At BMC, research is always in progress.

Learn more biomedcentral.com/submissions 